\title{
Participación paterna en la experiencia del parto
}

\author{
H. Villalón ・ R. Toro・I. Riesco・M. Pinto・C. Silva
}

\begin{abstract}
Father involvement in childbirth

Introduction: Recent initiatives have promoted the participation of fathers in the early care of their children. Objective: To assess the results of a program to encourage parental involvement in childbirth. Parents of healthy term newborns were randomly allocated to participate either in the birth experience or control. Patients and Methods: The protocol included: to dry the skin, umbilical cord cutting off, weight, height, and finally give him/her to the mother for the skin-to-skin contact. Heart rate (HR), respiratory (RR) and temperature were evaluated one hour later. In the first outpatient clinic assessment, mothers completed a questionnaire. 127 fathers participated either in the birth experience or control. Results: 62 followed the protocol and 65 the control. Both newborn groups were comparable. Also were fathers in age, education and rurality; mothers in primiparity. Significant differences: night care $(37 / 62,10 / 6559.6 \%$ vs $15.4 \%, \mathrm{p}<0.01)$; post prandial assistance $(50 / 62,14 / 6580.6 \%$ vs $21.5 \%$, p < 0.01); participation in bathing $(38 / 62,61.3 \%$ vs $15 / 65,23.1 \%, \mathrm{p}<0.01)$; newborn visit upon arrival at home $(46 / 62,74.2 \%$ vs $22 / 65,33.8 \%, \mathrm{p}<0.01)$; helping in crying episodes $(42 / 62,67.7 \%$ vs $17 / 65,26.1 \%, \mathrm{p}<0.01)$. There was stability in HR, RR and temperature one hour postpartum. Only one case of complication among parents (fainting). Conclusions: There were more cases of early care behaviors among participating fathers at birth, even belonging to a discouraging socio cultural environment. (Key words: Newborn, postnatal care, father involvement, family participation, fathering).

Rev Chil Pediatr 2014; 85 (5): 554-560
\end{abstract}

\section{RESUMEN}

Introducción: Recientes iniciativas, han promovido la participación de los padres en los cuidados tempranos de sus hijos. Objetivo: Evaluar los resultados de un programa de estímulo a la participación paterna en el parto. Se incluyeron padres de RN de término sanos, asignados aleatoriamente para participar en la experiencia del parto o control. Pacientes y Metodos: El protocolo incluyó: secado de la piel, corte de cordón umbilical, peso, estatura, y finalmente, entrega a la madre para el contacto piel a piel. Se evaluó frecuencia cardiaca (FC), respiratoria (FR) y temperatura una hora después. En el primer control ambulatorio, las madres completaron un cuestionario. 127 padres participaron en la experiencia del parto o control. Resultados: 62 asignados al

Recibido el 13 de septiembre de 2013, última versión aceptada para publicación el 28 de abril de 2014.

Hernán Villalón U. ( $\bowtie)$

Servicio de Neonatología, Departamento de Pediatría, Clínica Las Condes.

Facultad de Medicina. Universidad de Chile.

E-mail: hvillalon@clc.cl

Rosario Toro G., Isidora Riesco C.

Psicóloga, Servicio de Neonatología. Departamento de Pediatría, Clínica Las Condes.

Mauricio Pinto C., Cristián Silva V.

Servicio de Neonatología, Departamento de Pediatría, Clínica Las Condes. 
protocolo y 65 al control. Ambos grupos de RN resultaron comparables. También los padres, en edad, escolaridad, ruralidad, y las madres, en primiparidad. Diferencias significativas: asistencia nocturna $(37 / 62,59,6 \%$ vs $10 / 65,15,4 \%, \mathrm{p}<0,01)$; post prandial $(50 / 62,80,6 \%$ vs $14 / 65,21,5 \%, \mathrm{p}<0,01)$; participación en el baño (38/62, 61,3\% vs 15/65, 23,1\%, p < 0,01); visita al RN al llegar al domicilio (46/62, 74,2\% vs 22/65, 33,8\%, p $<0,01)$; ayuda ante episodios de llanto $(42 / 62,67,7 \%$ vs $17 / 65,26,1 \%, \mathrm{p}<0,01)$. Hubo estabilidad en FC, FR y temperatura una hora post-parto. Sólo un caso de complicación entre los padres (lipotimia). Conclusiones: Hubo más conductas de interés en cuidados tempranos, entre los padres participantes en el parto, aún perteneciendo a un medio sociocultural que no las promueve ni facilita.

(Palabras clave: Recién nacido, cuidado postnatal, participación paterna, participación familiar).

Rev Chil Pediatr 2014; 85 (5): 554-560

\section{Introducción}

En Chile, durante la última década, ha habido una creciente participación de los padres en los procesos de crianza de sus hijos, lo cual se ha visto favorecido por políticas públicas como "Chile crece contigo", en el cual se promueve la participación desde el embarazo y el nacimiento de sus hijos ${ }^{1}$. Previamente, el proceso del parto, en los centros de salud, estaba focalizado principalmente en los cuidados de la madre y el recién nacido, y considerado, por lo tanto, como un asunto de éstos y el sistema de salud, dejando así, muy poco espacio para la participación de los padres ${ }^{2}$. De esta manera, iría quedando atrás la visión tradicional, en países de nuestra región, en los cuales el rol paterno sería solamente de proveedor ${ }^{3}$, y reemplazado paulatinamente por un modelo de paternidad más cercano y participativo ${ }^{4}$.

Existe evidencia de los beneficios del contacto temprano de los padres con sus hijos recién nacidos, reportados en diversos estu$\operatorname{dios}^{5-9}$. Sin embargo, a nivel nacional, esto ha resultado difícil, por una sensación de incapacidad de responder ante la ausencia de referentes que puedan servir como modelos de interacción ${ }^{10}$, ya que de la experiencia de haber sido hijos ${ }^{11}$ y de lo aprendido a su padre o figura paterna en lo cotidiano, se busca la imitación o la diferenciación de los patrones de comportamiento parental ${ }^{12}$. Esto, también se ha reportado en otras comunidades de países en desarrollo ${ }^{13}$. Todo esto, podría explicar lo frecuentemente comentado por equipos médicos rurales de la región de Aysén, hasta hace algunos años, en relación a la poca par- ticipación de los padres en la crianza de sus hijos durante los primeros meses de vida (observaciones no publicadas). Como apoyo al desarrollo de las políticas, tipo "Chile crece contigo", en los cuales se fomenta el acompañamiento paterno durante el parto, quisimos presentar esta experiencia, que si bien se realizó en los años 1999 y 2000 -cuando aún la presencia de la pareja en dicho momento no era habitual-, podría ser un aporte a la implementación de estrategias para promover la participación del padre en los cuidados tempranos de sus hijos.

El objetivo de este estudio es evaluar una estrategia de participación paterna en la experiencia del parto, para promover el interés en cuidados temprano de sus hijos.

\section{Pacientes y Método}

\section{Diseño}

Estudio clínico, prospectivo y controlado con selección aleatoria, realizado en la Maternidad del Hospital Regional de Coyhaique, durante un período de 23 meses, durante los años 1999 y 2000 . Se enrolaron 133 padres, de manera consecutiva, y que contaban con los criterios de inclusión, en el momento en que sus mujeres fueron ingresadas a pre-parto. De este modo, los participantes pares fueron asignados para participar en la experiencia del parto (grupo A) y los impares al control (grupo B). Seis de los 68, del grupo A se negaron a participar luego de ser informados, consintiendo $62(91,2 \%)$. Se les invitó a asistir a la sala de partos para acompañar a sus esposas, y a seguir un protocolo bajo supervisión del equipo 
médico: secar la piel de sus bebés, cortar el cordón umbilical, sostenerlos, pesarlos, medirlos, y finalmente entregarlos a las madres para establecer el contacto piel a piel. Una hora después del parto, se evaluó temperatura, frecuencias cardíaca y respiratoria a los niños. En los padres, se registró la ocurrencia de lipotimia o malestar. El grupo control, de acuerdo a la metodología de esa época, tuvo contacto con sus mujeres e hijos, una vez en las salas de la maternidad. Se incluyó RN de término, sanos, con puntuación de apgar mayor a 8 (1-5 min). Otros criterios de exclusión: macrosomía, madre diabética, riesgo de estreptococo grupo B, fiebre durante el trabajo de parto, menos de $3.000 \mathrm{~g}$ al nacer y parejas sin convivencia estable.

\section{Seguimiento}

En el primer control ambulatorio, se solicitó a las madres completar un cuestionario en ambiente confidencial, para evaluar diferentes parámetros referentes a la participación del padre en el hogar (tabla 1): asistencia post prandial y nocturna del RN, participación en el procedimiento de baño, ayuda ante el llanto, cambio de pañales, visita del RN como primera actividad al llegar al domicilio, y mejoría en la relación de pareja. Las evaluaciones fueron cualitativas (Sí/No). Además, se registró la concurrencia del padre a la primera consulta ambulatoria.

\section{Análisis estadístico}

Los datos fueron analizados con Epi Info 7. Prueba $t$ de Student para resultados paramétricos y el análisis $\chi^{2}$ para proporciones y resultados no paramétricos. Se consideró significativo, valores inferiores al 5\%. Este manuscrito contó con la aprobación por el Comité de Ética institucional.

\section{Resultados}

Los resultados globales se muestran en la tabla 2. Ambos grupos fueron comparables en peso $(3.390 \pm 348$ vs $3.421 \pm 335 \mathrm{~g}$, NS) y género. Así como también, lo eran los padres en edad $(27,4 \pm 3,4$ vs $26,1 \pm 2,6$ años, NS), escolaridad completa $(46 / 62,74,2 \%$ vs $48 / 65,73,8 \%$, NS), ruralidad (29/62, el 46,8\% vs $29 / 65,40,0 \%$, NS) y primiparidad de las madres (24/62, 38,7\% vs 20/65, 30,7\%, NS).

No se observaron diferencias significativas en los parámetros fisiológicos evaluados una hora después del parto: temperatura $(36,7 \pm 0,4$ ${ }^{\circ} \mathrm{C}$ vs $36,5 \pm 0,14^{\circ} \mathrm{C}$, NS), frecuencia cardíaca $(146 \pm 7,4$ vs $146 \pm 12,8 \mathrm{lpm}, \mathrm{NS})$ y frecuencia respiratoria $(47,1 \pm 4,4 v s$ 45,7 $\pm 6,3$, NS $)$. Tampoco en días de hospitalización (en ambos grupos la mediana fue 2 días), ni en lactancia materna exclusiva $(87,1 \%, 54 / 62$ vs $83,1 \%$, $54 / 65$, NS).

Las principales diferencias entre ambos grupos se registraron en la colaboración durante las noches y después de la alimentación $(37 / 62,59,6 \%$ vs $10 / 65,15,4 \%, \mathrm{p}<0,0001$, y $50 / 62,80,6 \%$ vs $14 / 65,21,5 \%, \mathrm{p}<0,0001)$, en la participación en el baño (38/62, 61,3\% vs $15 / 65,23,1 \%, \mathrm{p}<0,0001)$, visita al recién nacido al llegar a la casa $(46 / 62,74,2 \%$ vs $22 / 65,33,8 \%, \mathrm{p}<0,0001)$, ayuda ante el llanto del RN (42/62, 67,7\% vs 17/65, 26,1\%, $\mathrm{p}<0,0001)$ y asistencia del padre a la primera evaluación clínica ambulatoria $(51,6 \%, 32 / 62$ vs $21,5 \%, 14 / 65, \mathrm{p}<0,001)$.

Además, las madres señalaron mejoría en la relación de pareja $(56,5 \%, 35 / 62$ vs $20,0 \%$, $13 / 65, \mathrm{p}<0,001)$. No se observaron diferencias en el cambio de pañales $(24,2 \%, 15 / 62 v s$ $18,5 \%, 12 / 65$, NS). Estos resultados se muestran en las figuras 1 y 2 . Sólo fue registrado un caso de lipotimia entre los padres como complicación $(1,6 \%)$.

Tabla 1. Encuesta realizada a las madres en el primer control neonatal ambulatorio

Sí/No

Cuándo su hijo despierta llorando en la noche, el padre se levanta a ayudarlo?

Después de tomar pecho, el padre lo ayuda a eliminar los gases?

Ayuda el padre a bañar a su hijo?

Cuando su hijo está llorón e irritable, lo toma el padre en brazos para calmarlo?

Cuando el padre está en la casa, cambia los pañales de su hijo?

La primero que hace el padre al llegar a la casa, es ir a ver al bebé?

Encuentra Ud, que su relación de pareja ha mejorado después del parto? 
Tabla 2. Características de los padres en relación a participación en el parto de sus hijos $(\mathbf{n}=127)$

\begin{tabular}{|c|c|c|c|}
\hline & Participa (+) & Participa ( - ) & Valor p \\
\hline Masculino & $48,4 \%(30 / 62)$ & $44,6 \%(29 / 65)$ & NS \\
\hline Femenino & $51,6 \%(32 / 62)$ & $55,4 \%(36 / 65)$ & NS \\
\hline Peso & $3.390 \pm 348$ & $3.421 \pm 335$ & NS \\
\hline $\mathrm{T}^{\circ}$ & $36,7 \pm 0,4$ & $36.5 \pm 0,14$ & NS \\
\hline FC & $146 \pm 7,4$ & $146 \pm 12,8$ & NS \\
\hline FR & $47,1 \pm 4,4$ & $45,7 \pm 6,3$ & NS \\
\hline Días hosp (mediana) & $2(2-3)$ & $2(1-4)$ & \\
\hline Primiparidad & $38,7 \%(24 / 62)$ & $30,7 \%(20 / 65)$ & NS \\
\hline Edad paterna & $27,4 \pm 3,4$ & $26,1 \pm 2,6$ & NS \\
\hline Escolaridad completa & $74,2 \%(46 / 62)$ & $73,8 \%(48 / 65)$ & NS \\
\hline Ruralidad & $46,8 \%(29 / 62)$ & $40,0 \%(26 / 65)$ & NS \\
\hline LME & $87,1 \%(54 / 62)$ & $83,1 \%(54 / 65)$ & NS \\
\hline Cambio pañales & $24,2 \%(15 / 62)$ & $18,5 \%(12 / 65)$ & NS \\
\hline Asistencia nocturna & $59,6 \%(37 / 62)$ & $15,4 \%(10 / 65)$ & $<0,0001$ \\
\hline Asist. post prandial & $80,6 \%(50 / 62)$ & $21,5 \%(14 / 65)$ & $<0,0001$ \\
\hline Baño & $61,3 \%(38 / 62)$ & $23,1 \%(15 / 65)$ & $<0,0001$ \\
\hline Relación de pareja mejor & $56,5 \%(35 / 62)$ & $20 \% \quad(13 / 65)$ & $<0,001$ \\
\hline Visita RN al llegar & $74,2 \%(46 / 62)$ & $33,8 \%(22 / 65)$ & $<0,0001$ \\
\hline Asistencia al llorar & $67,7 \%(42 / 62)$ & $26,1 \%(17 / 65)$ & $<0,0001$ \\
\hline Asist. paterna $1^{\circ}$ control & $51,6 \%(32 / 62)$ & $21,5 \%(14 / 65)$ & $<0,001$ \\
\hline
\end{tabular}
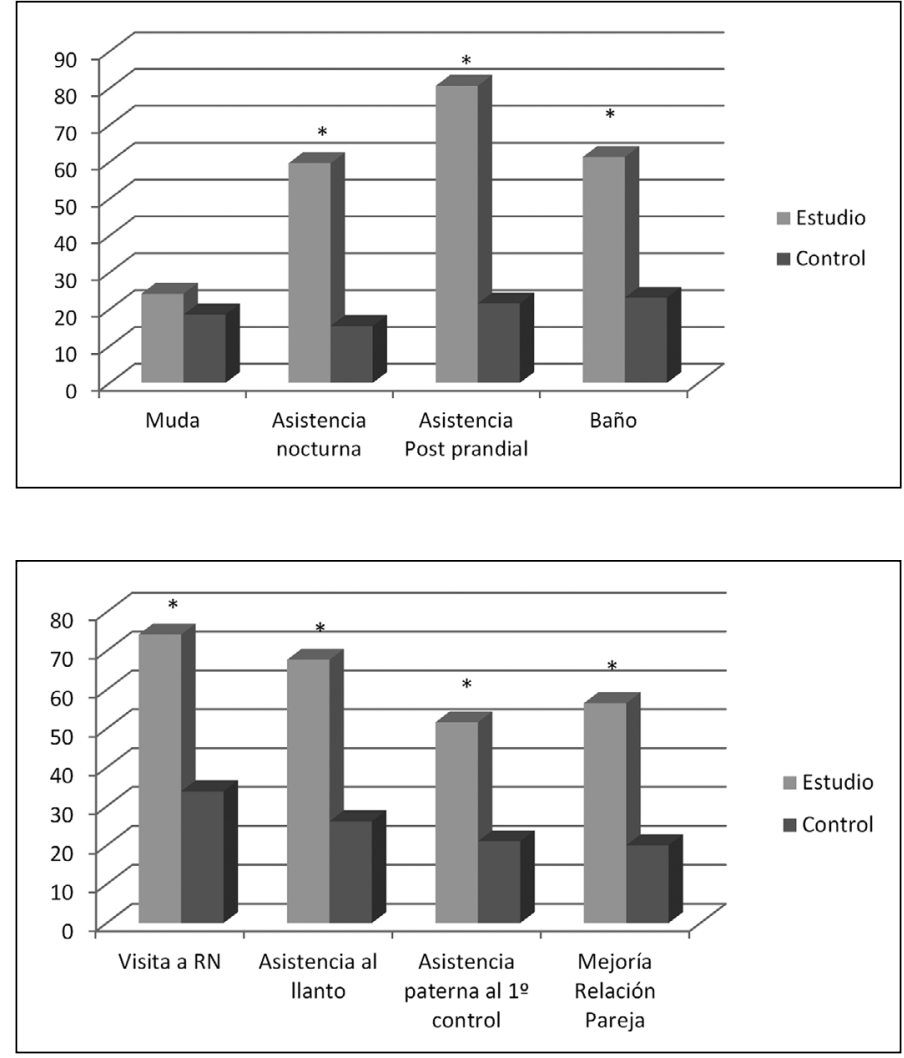

Figura 1. Parámetros de cuidados paternos a hijos recién nacidos $(n=127) . * p<0,01$

Figura 2. Parámetros de cuidados paternos a hijos recién nacidos $(n=127)$. ${ }^{*} \mathrm{p}<0,01$. 


\section{Discusión}

Este estudio demostró que ambos grupos son comparables, en las características de los padres participantes, recién nacidos y paridad de las madres. La metodología sería segura para los niños, al considerar la estabilidad de los parámetros fisiológicos, en términos de termorregulación, frecuencia cardíaca y respiratoria. Así mismo, es bien tolerada y aceptada, por los padres participantes. En esta serie no se reportaron efectos adversos significativos, descontrol u otros, salvo un caso de lipotimia leve sin caída al piso.

Esta es una experiencia realizada hace muchos años, que consideramos novedosa por la metodología misma, ya que más allá del acompañamiento de sus parejas, implicó una participación directa y protagónica de los padres en el nacimiento de sus hijos, al interior de las salas de parto. Para evaluar los efectos conductuales en ellos, no se utilizó una escala validada, sino que una encuesta aplicada a las madres, referente a conductas cotidianas de cuidados de los niños recién nacidos en sus hogares, y a la percepción subjetiva de su relación de pareja. Sí, se tuvo la precaución de aplicarla en condiciones de confidencialidad. Así mismo, en la época de realización de esta experiencia, la asistencia de los padres a los partos resultaba absolutamente inhabitual y ajena a la idiosincrasia del lugar, lo que la hizo particularmente innovadora.

Se observaron diferencias significativas, en muchos aspectos, que pudieran implicar conductas de interés en la atención de sus hijos, por parte de los padres participantes en la experiencia del parto. Entre éstas, destacan la asistencia post-prandial (ayudar en la eliminación de gases deglutidos), al llanto y nocturna, la ayuda en el procedimiento de bañar al recién nacido y la visita inmediata al momento de llegar después del trabajo. Llama la atención, la falta de participación en el cambio de pañales, posiblemente, por factores de tipo cultural. Un hallazgo interesante, referido por las madres, es la mejoría en la relación de pareja, en el grupo estudiado en relación al control. La metodología no incidiría en la lactancia ni en el tiempo de hospitalización, sino más bien, en la relación del padre con su hijo/hija recién nacido y cónyuge, en un medio social y cultural que no promueve ni facilita dichas conductas. Así, el acompañamiento por parte de la pareja contribuye a fortalecer los vínculos entre los miembros de ésta, y es una oportunidad particularmente relevante para la relación con su hijo o hija que está naciendo, y con las responsabilidades de la paternidad ${ }^{1}$, esto se ha ido promoviendo de manera más activa en nuestro país en los últimos años ${ }^{14}$.

Revisiones sistemáticas, han evidenciado la importancia de la interacción padre-hijo, en un pronóstico de largo plazo más favorable en los aspectos sociales, psicológicos y conductuales ${ }^{5}$, así como la positiva percepción del padre por parte de su propio hijo, al existir una interacción efectiva ${ }^{15}$. Experiencias en otros países de la región, han mostrado un mayor compromiso de los padres con los procesos de crecimiento y desarrollo de sus hijos ${ }^{6}$.

La vivencia de una experiencia compleja y diferente, por parte de los padres, podría haber influido en el cambio de percepción hacia el parto, lo que podría haber contribuido a un mayor respeto y admiración hacia sus parejas ${ }^{16}$. Esto podría explicar lo referido en la encuesta, relativo a una mejor relación entre ellos.

También en la literatura, se reportan otros beneficios de este contacto temprano padrehijo. Al realizar un contacto piel a piel, en partos cesáreas, los recién nacidos lloraron menos y lograron un estado relajado antes que en el grupo control ${ }^{7,8}$, además, presentaron patrones de succión más precoces ${ }^{8} \mathrm{e}$ intentos de comunicación vocalizada ${ }^{7}$. Padres que sostuvieron en brazos a sus hijos, durante la primera hora de vida, presentaron un mayor comportamiento no verbal hacia ellos, al evaluar al mes con la escala de evaluación neonatal de Brazelton (BNAS) ${ }^{9}$. De esta manera, el padre debiera ser el primer cuidador para su hijo recién nacido, en caso de separación con su madre ${ }^{8}$.

En nuestro estudio, no se observaron diferencias en la colaboración paterna en el cambio de pañales, lo que se podría explicar por una influencia cultural. Eso también se ha concluido en otros reportes, en los que se plantea que la transición a la paternidad está muy 
influenciada por el contexto social y laboral ${ }^{17}$. Si bien es cierto, podría considerarse que la asistencia al parto de su propio hijo es el inicio de la paternidad ${ }^{18}$, este no resulta fácil, y muchos padres la consideraron emocionalmente abrumadora al vivirla sin una preparación previa $^{17,19}$, y esta misma falta de conocimiento los lleva a evitarla ${ }^{18}$. En esto, los equipos de salud, de diferentes culturas, reconocen la necesidad de aumentar el compromiso de los hombres con los cuidados maternales de sus parejas e hijos recién nacidos ${ }^{1,6,13,14}$. En nuestro país, este ha sido uno de los objetivos de la política "Chile crece contigo". Al sentirse estimulados y apoyados por los profesionales de las maternidades, la mayoría de los padres, en diferentes experiencias, la refieren como positiva $^{1,20}$. Se hace necesaria una discusión social amplia, que involucre a los servicios médicos, que amplíe aún más y asegure la presencia y participación de los padres en el trabajo de parto de sus mujeres y nacimiento de sus hijos ${ }^{1,2,14}$.

Esta es una experiencia realizada hace años, en la región de Aysén, aunque antigua $\mathrm{y}$ con las limitaciones propias de una muestra relativamente pequeña, pensamos interesante de mostrar, ya que podría ser un aporte a los equipos de salud de países en desarrollo, y a nuestra política nacional "Chile crece contigo", como estrategia para promover conductas de acercamiento a los cuidados tempranos de los niños y como apoyo a las madres, por parte de los padres.

En conclusión, la experiencia expuesta en este trabajo, pareciera ser exitosa, en el sentido de generar conductas de interés en los cuidados tempranos hacia sus hijos recién nacidos, por parte de padres pertenecientes a un medio social y cultural que no promovía ni facilitaba dichas conductas. Esto debiera motivar futuros estudios, tendientes a promover una mayor participación de los padres, incluso, desde el momento mismo del parto.

Potenciales conflictos de interés: Este trabajo cumple con los requisitos sobre consentimiento /asentimiento informado, comité de ética, financiamiento, estudios animales y sobre la ausencia de conflictos de intereses según corresponda.

\section{Referencias}

1.- Aguayo F, Kimelman E, Correa P: Estudio sobre la Participación de los Padres en el Sistema Público de Salud de Chile. Informe Final. Chile: CulturaSalud/MINSAL, 2012. http://chccsalud.cl/archivos/estudios/Estudios/ Paternidad/202012.pdf

2.- Olavarría J: Los hombres también somos fecundos. En: Olavarría J y Márquez A ed. Varones: Entre lo público y la intimidad. IV Encuentro de estudios de masculinidades. Santiago, Chile: FLACSO, 2004.

3.- Olavarría J: Ser padre en Santiago de Chile. En: N. Fuller ed. Paternidades en América Latina. Lima, Ed. Pontificia Universidad Católica del Perú, Fondo Editorial, 2000; 129-73.

4.- Fuller N: Introducción. En N. Fuller ed. Paternidades en América Latina. Lima, Ed. Pontificia Universidad Católica del Perú, Fondo Editorial 2000; 11-32.

5.- Sarkadi A, Kritiansson R, Oberklaid F, Bremberg S: Fathers' involvement and children's developmental outcomes: a systematic review of longitudinal studies. Acta Paediatr 2008; 97 (2):153-8.

6.- De Carvalho $M L$ : Fathers' participation in childbirth at a public hospital: institutional difficulties and motivations of couples. Cad Saude Publica 2003; 19 Suppl 2: S389-98.

7.- Velandia M, Mathisen AS, Uvnäs-Moberg K, Nissen E: Onset of vocal interaction between parents and newborns in skin-to-skin contact immediately after elective cesarean section. Birth 2010; 37 (3): 192-201.

8.- Erlandsson K, Fagerberg I, Christensson K: Skin-toskin care with the father after cesarean birth and its effect on newborn crying and prefeeding behavior. Birth 2007; 34 (2): 105-14.

9.- Jones $C$ : Father to infant attachment: effects of early contact and characteristics of the infant. Res Nurs Health 1981; 4 (1): 193-200.

10.- Montecino $S$ : Nuevas feminidades y masculinidades. Una mirada de género al mundo evangélico de La Pintana. Estudios Públicos 2002; 87: 73-103.

11.- Cruzat C, Aracena M: Significado de la paternidad en jóvenes varones del sector sur-oriente de Santiago. Psykhe 2006; 15: 29-44.

12.- Olavarría J: Y todos querían ser buenos padres. Varones de Santiago de Chile en conflicto. Santiago: FLACSO, 2001.

13.- Iliyasu Z, Galadanci HS, Aliyu MH: Birth preparedness, complication readiness and fathers' participation in maternity care in a northern Nigerian community. Afr. J. Reprod. Health 2010; 14 (1): 21-32. 
14.- Aguayo F, Sadler M: El papel de los hombres en la equidad de género: ¿qué masculinidades estamos construyendo en las políticas públicas de Chile? En: Aguayo, F. y Sadler, M. (eds) Masculinidades y políticas públicas. Involucrando hombres en la equidad de género. Universidad de Chile/CulturaSalud/EME. 2011.

15.- Magill-Evans J, Harrison MJ, Rempel G, Slater L: Interventions with fathers of young children: systematic literature review. J Adv Nurs 2006; 55 (2): 248-64.

16.- Chandler $S$, Field PA: Becoming a father. First-time fathers' experience of labor and delivery. J Nurse Midwifery 1997; 42 (1): 17-24.
17.- Genesoni L, Tallandini MA: Men's psychological transition to fatherhood: an analysis of the literature, 19892008. Birth 2009; 36 (4): 305-18.

18.- Longworth HL, Kingdon CK: Fathers in the birth room: what are they expecting and experiencing? A phenomenological study. Midwifery 2011; 27 (5): 588-94.

19.- Sapkota S, Kobayashi T, Takase M: Husbands' experiences of supporting their wives during childbirth in Nepal. Midwifery 2012; 28 (1): 45-51.

20.- Hildingsson I, Cederlöf L, Widen S: Fathers' birth experience in relation to midwifery care. Women Birth 2011; 24 (3): 129-36. 